Zusammenfassung der Valenzen $5-5^{\prime}$ und $6-6^{\prime}$ :

$$
x_{\mu}=+0,4654 \quad y_{\mu}=0 \quad z_{\mu}=-1,6684 .
$$

c) Die Valenzen $7_{\mathrm{s}}^{\prime}$ und $7_{\mathrm{a}}{ }^{\prime}(s . A b b .3)$

$$
\begin{aligned}
\operatorname{tg} \beta & =\frac{z_{7}-z_{1}}{x_{7}-x_{1}}, \quad \beta=56,21^{\circ} . \\
\alpha_{7_{s}^{\prime}} & =110,95^{\circ}, \\
\alpha_{7_{\mathrm{a}}^{\prime}} & =1,47^{\circ},
\end{aligned}
$$

Die Einheitsvektoren:

$$
\begin{array}{llll}
7_{\mathrm{s}}^{\prime} & x_{\mu}=-0,3574 & y_{\mu}=0 & z_{\mu}=+0,9339 \\
7_{\mathrm{a}}^{\prime} & x_{\mu}=+0,9997 & y_{\mu}=0 & z_{\mu}=+0,0257 \\
\cline { 2 - 4 } & x_{\mu}=+0,6423 & y_{\mu}=0 & z_{\mu}=+0,9596
\end{array}
$$

Zusammengefaßt ergeben sich für die $6 \mathrm{C}-\mathrm{Cl}$-Bindungen des Hexachlorbicycloheptens folgende Vektor- komponenten :

$$
\begin{array}{lll}
1^{\prime}+4^{\prime} & x_{\mu}=+0,4892 & z_{\mu}=+0,5148 \\
5^{\prime}+6^{\prime} & x_{\mu}=+0,4654 & z_{\mu}=-1,6684 \\
7 \mathrm{~s}^{\prime}+7 \mathrm{a}^{\prime} & x_{\mu}=+0,6423 & z_{\mu}=+0,9596 \\
& x_{\mu}=+1,5969 & z_{\mu}=-0,1940
\end{array}
$$

Mit Benutzung der Bindungsmomente:

$\mu_{\mathrm{B}}=2,15 \mathrm{D}$ für die $\mathrm{C}-$ Cl-Valenzen $\mathrm{I}^{\prime}+4^{\prime}$ (aus den Momenten von tert.-Butyl- und tert.-Amylchlorid), $\mu_{\mathrm{B}}=1,40 \mathrm{D}$ für die C-Cl-Valenzen $5^{\prime}+6^{\prime}$ (aus den Momenten von cis-2.3-Dichlor-buten-(2), o-Dichlorbenzol und 2.3-Dichlornaphthalin) und

$\mu_{\mathrm{B}}=1,95 \mathrm{D}$ für die $\mathrm{C}-\mathrm{Cl}$-Valenzen $7{ }_{\mathrm{s}}{ }^{\prime}+7_{\mathrm{a}}{ }^{\prime}$ (aus den Momenten von 2.2-Dichlorpropan und 1.1-Dichlorcyclopentan)

ergibt sich für das 1.4.5.6.7.7-Hexachlor-bicyclo[2.2.1]-hepten-(5) (I) ein Gesamtmoment von 3,02 D, das in guter Übereinstimmung mit gemessenen Werten ${ }^{5,6}$ steht.

\title{
Considerations of image contrast in Electron Microscopy of objects composed of low atomic number
}

\author{
By M. L. DE \\ Biophysics Division, Saha Institute of Nuclear Physics, Calcutta-9, India \\ (Z. Naturforschg. 17 b, 728-731 [1962] ; eingegangen am 17. März 1962)
}

\begin{abstract}
Defects of the electron scattering formula based on Thomas-Fermi atom model and of L e is e ga n g's formula, are first briefly discussed in relation to image contrast problem in Electron Microscopy. A few aspects of the electron scattering formula of $\mathrm{Lenz}$ are then considered particularly for dealing with elements of low atomic number. From a consideration of this formula it is shown that the image contrast of a given mass thickness of object is liable to be dependent on the atomic number of the object.
\end{abstract}

It is well known that contrast in the electron microscope image of an object, arises due to differential scattering of the beam electrons in the different parts of the object. The question of electron scattering and image contrast resulting therefrom, have been studied both theoretically as well as experimentally by several authors. The earlier work of $\mathrm{S}_{\mathrm{CHIFF}}{ }^{1}$, Boersch ${ }^{2}$ and Borries ${ }^{3}$ were based on Th o m a s-F e r mi atom model and Borries particulary, by using the elastic and inelastic scattering formula of MoLiere ${ }^{4}$ and $\mathrm{Koppe}^{5}$ respectively, had come to a number of interesting conclusions. But in the electron microscopy of biological samples, one naturally deals with matter composed of low atomic

1 L. I. Schiff, Physic. Rev. 61, 72 [1942].

2 H. Boersch, Z. Naturforschg. 2 a, 615 [1947].

3 B. V. Borries, Z. Naturforschg. 4 a, 51 [1949].

${ }^{4}$ G. Moliere, Z. Naturforschg. 2 a, 133 [1947]. weight elements for which the $\mathrm{TF}$ statistical model does not provide a good representation particularly in the small angle region ( $\sim 10^{-2}$ radian) of electron microscopic work. Further, the experimental results of Leonhard ${ }^{6}$ and Coupland ${ }^{7}$ also showed the invalidity of the Molier e-Koppe formulae for low as well as for high atomic weight samples. LeIsEgang $^{8}$, after first B or n approximation, had derived electron scattering formula for multiple elastic collisions, and showed good agreement of the formula with his experimental results at fairly large scattering angles for comparatively thick samples of $\mathrm{Au}(150 \AA), \mathrm{Al}(270 \AA)$ and $\mathrm{Ag}$

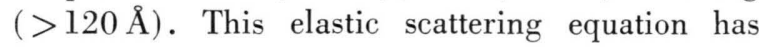

5 H. Koppe, Z. Physik 124, 658 [1947].

6 F. Leonhard, Z. Naturforschg. 9 a, 1019 [1954].

7 J. H. Coupland, Proc. physic. Soc. 69 B, 46 [1956].

8 S. Leisegang, Z. Physik 132, 183 [1952]. 
been used by several authors ${ }^{9-13}$ for estimation of mass thickness of biological substances by measurement of contrast of electron microscope image. In these cases, however, the inelastic scattering crosssection has been totally disregarded although in the small angle region of electron microscope work, the inelastic scattering is more predominant than the elastic scattering process.

Lenz ${ }^{14}$, by making use of the elastic and inelastic scattering equations of Wentzer ${ }^{15}$ and Morse ${ }^{16}$, developed scattering formula based on $\mathrm{Har} \mathrm{tree}$ atom model; and they were therefore very suitable for low atomic weight elements and for small scattering angle. Lenz's equations made satisfactory agreement with the earlier experimental results of Biberman et al. ${ }^{17}$ and with the subsequent works of other authors ${ }^{18-20}$; further, Silvester and Burge 21 on the basis of these equations, computed for biological matter, the 'aufhellungsdicke', which denotes the upper limit of mass thickness which can be usefully examined with the electron microscope.

Sadhukhan ${ }^{22}$ made certain modifications in the L e $\mathrm{n}$ z's equations by considering the atomic radius on Wentzel model $\left(R=a_{\mathrm{H}} \cdot Z^{-1 / 3}\right)$; and these modified equations were used by CossLEtT ${ }^{23}$ in his paper dealing with quantitative electron staining.

In the present paper some aspects of Lenz's equations have been considered particularly for elements of low atomic weight, showing the nature of dependence of the scattering cross-sections of the various atoms on their atomic numbers. Considerations are then given to the resulting image contrast expected on the basis of these scattering crosssections.

\section{Consideration of Lenz's Scattering Formula}

According to the equation of $\mathrm{Lenz}$, the expressions for the differential cross-section of an atom (at. No. Z), for elastic and inelastic scattering, can be writ-

9 E. Zeitler and G. F. Bahr, Exp. Cell Res. 12, 44 [1957].

10 M. L. De and P. Sadhukhan, Nature [London] 182, 1008 [1958].

11 E. Zeitler and G. F. Bahr, J. appl. Physics 30, 940 [1959].

12 G. Bloom and E. Zeitler, Exp. Cell Res. 17, 13 [1959].

13 L. H. Sobin, G. Bloom and E. Zeitler, Exp. Cell Res. 17, 472 [1959].

14 F. Lenz, Z. Naturforschg. 9 a, 185 [1954].

15 G. Wentzel, Z. Physik. 40, 590 [1927].

16 P. M. Morse, Physik. Z. 33, 443 [1932].

17 L. M. Biberman, E. N. Wrorow, I. A. Kowner, N. G. ten as,

$$
\begin{aligned}
\frac{\mathrm{d} \sigma_{\mathrm{e}}}{\mathrm{d} \Omega} & =\frac{4 Z^{2}}{a_{\mathrm{H}}^{2}} \frac{1}{\left(q^{2}+\frac{1}{R^{2}}\right)^{2}} \\
\frac{\mathrm{d} \sigma_{\mathrm{in}}}{\mathrm{d} \Omega} & =\frac{4 Z}{a_{\mathrm{H}}^{2} q^{4}}\left[1-\frac{1}{\left(q^{2} R^{2}+1\right)^{2}}\right]
\end{aligned}
$$$$
\text { with } \quad q=\frac{4 \pi}{\lambda} \cdot \sin \frac{a}{2}
$$

where $a_{\mathrm{H}}$ is the $\mathrm{B} o \mathrm{~h} \mathrm{r}$ radius of the Hydrogen atom, $\lambda$ is the $\mathrm{de}-\mathrm{Brogli}$ e electron wavelength, $\alpha$ is the scattering angle, and $R$ is the screening radius of the atom. $R$ has been represented by Lenz as

$$
R=(\Theta / 6 Z)^{1 / 2}
$$

where the parameter $\Theta$ is related to the electron density distribution in the atomic shell. $\Theta$ has been expressed as $\Theta=k \cdot a_{\mathrm{H}}{ }^{2}$ where $k$ is a numerical factor, values of which for several atoms on $\mathrm{Hartr}$ e e model have been tabulated by WYrwich and Lenz ${ }^{24}$. With these tabulated values of $\Theta$ combined with equation (4) one gets values of the screening radius of several atoms as represented in Fig. 1, here for comparison, the corresponding $\mathrm{Wentzel}$ radiuses have also been shown. It is seen that while the $\mathrm{Wentzel}$ radius decreases smoothly with increase of $Z$, the $\mathrm{H}$ artree radius is a discontinuous function of $Z$.

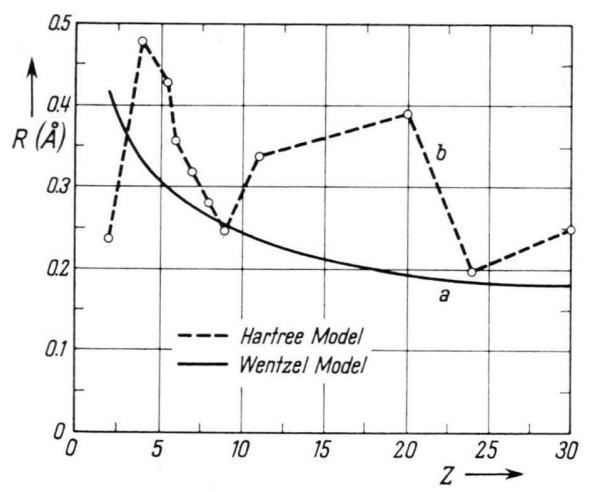

Fig. L. Variation of the screening radius of atoms with Atomic Number $Z$, calculated on $\mathrm{Hartree}$ and $\mathrm{Wentzel}$ atom models.

Fig. 2 shows the angular variation of differential cross-section of carbon for elastic scattering calculated

Sruschkin and B. M. Jaworskit, C. R. [Doklady] Acad. Sci., U.R.S.S. 69, 519 [1949].

18 G. KempF and F. Lenz, Proc. Stockholm Conf. Electron Microscopy, p. 67, Almquist and Wiksell, Stockholm 1957. 19 C. E. Hall and T. Inoue, J. appl. Physics 28, 1348 [1957].

20 M. E. Haine and A. W. Agar, Brit. J. appl. Physics 10, 341 [1959].

21 N. R. Silvester and R. E. Burge, Nature [London] 188, 641 [1960].

22 P. Sadhukhan, J. appl. Physics 29, 1235 [1958].

23 V. E. Cosslett, J. Roy. microscop. Soc. 78, 18 [1958].

24 H. W YrWich and F. Lenz, Z. Naturforschg. 13 a, 515 [1958]. 
on Hartree and Wentzel models with Lenz's equations and on TF model with Molier e's equation. It is seen that in the angular range of $10^{-4}$ to $10^{-2}$ radian and for $50 \mathrm{kV}$ electrons, the $\mathrm{H}$ a $\mathrm{rtree}$ cross-section is about 2.5 times greater than the $\mathrm{W}$ entzel value and about 4 times smaller than $\mathrm{TF}$ cross-section.

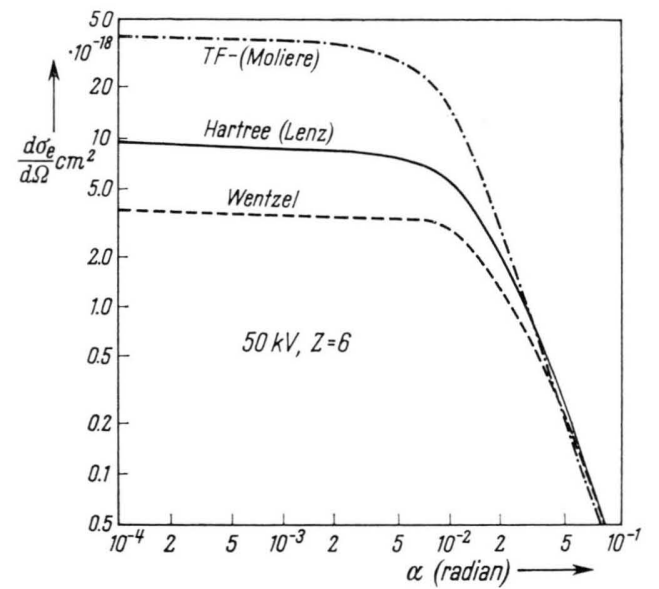

Fig. 2. Variation of the differential elastic scattering crosssection of carbon atom with angle, for $50 \mathrm{kV}$ electrons, calculated on the basis of TF, Hartree and Wentzel atom models.

The expressions for the total elastic and inelastic cross-section can be written after suitable transformation as in the Table below.

\begin{tabular}{|c|c|c|}
\hline $\begin{array}{l}\text { Total scattering } \\
\text { cross-section }\end{array}$ & Hartree & $\mathrm{W}_{\text {ENTZEL }}$ \\
\hline $\begin{array}{c}\text { Elastic: } \\
\left(\sigma_{\mathrm{e}}\right)_{0}^{\pi}=\int_{0}^{\pi} \mathrm{d} \sigma_{\mathrm{e}} \\
\text { Inelastic: } \\
\left(\sigma_{\mathrm{in}}\right)_{q}^{\pi}=\int_{q}^{\pi} \mathrm{d} \sigma_{\mathrm{in}} \\
\sigma_{\mathrm{in}} \\
\sigma_{\mathrm{e}}\end{array}$ & $\begin{array}{c}0.106 k \lambda^{2} \ln \frac{6 Z}{q^{2} k \frac{a_{\mathrm{H}}^{2}}{2}} \\
\frac{2}{Z} \ln \frac{6 Z}{q^{2} k a_{\mathrm{H}}^{2}}\end{array}$ & $\begin{array}{c}0.632 \lambda^{2} Z^{1 / 3} \ln \frac{Z^{2 / 3}}{q^{2} a_{\mathrm{H}}^{2}} \\
\frac{2}{Z} \ln \frac{Z^{2 / 3}}{\bar{q}^{2} a_{\mathrm{H}}^{2}}\end{array}$ \\
\hline
\end{tabular}

Table I. Total scattering cross-sections on Hartree and Wentzel Models.

In the above expressions, the parameter $q$ denotes the minimum inelastic scattering angle and is given by

$$
q=\frac{2 \pi}{\lambda} \frac{\Delta E}{U}
$$

where $\Delta E$ is the ionisation potential of the atom and $U$ is the electron energy, both in volts. From the tabulated values of $\Delta E$ for the various atoms as is given by Landolt and Bonnstein ${ }^{25}$ one finds that, for $U$ in the

25 Landolt and Bornstein, Zahlenwerte und Funktionen, 1, 1, 211, 6. Auflage, 1950. range of $50-100 \mathrm{kV}, \ln q$ is practically constant, so that both $\sigma_{\mathrm{e}}$ and $\sigma_{\text {in }}$ can be plotted respectively as $\sigma_{\mathrm{e}} / \lambda^{2}$ and $\sigma_{\text {in }} / \lambda^{2}$ against $Z$, as shown in Fig. 3 . Here curves (1) and (2) are drawn on $\mathrm{H}$ a r tre e model and curves $1(\mathrm{~A})$ and $2(\mathrm{~A})$ on $\mathrm{W}$ e n t z e l model. The ratio $\left(\sigma_{\mathrm{in}} / \sigma_{\mathrm{e}}\right)$ for the various atoms has been found to be practically the same on the two models, and curve 3 in Fig. 3 represents the said ratio, while curve 3 (A) has been taken from Fig. 7 of Lenz's paper ${ }^{14}$.

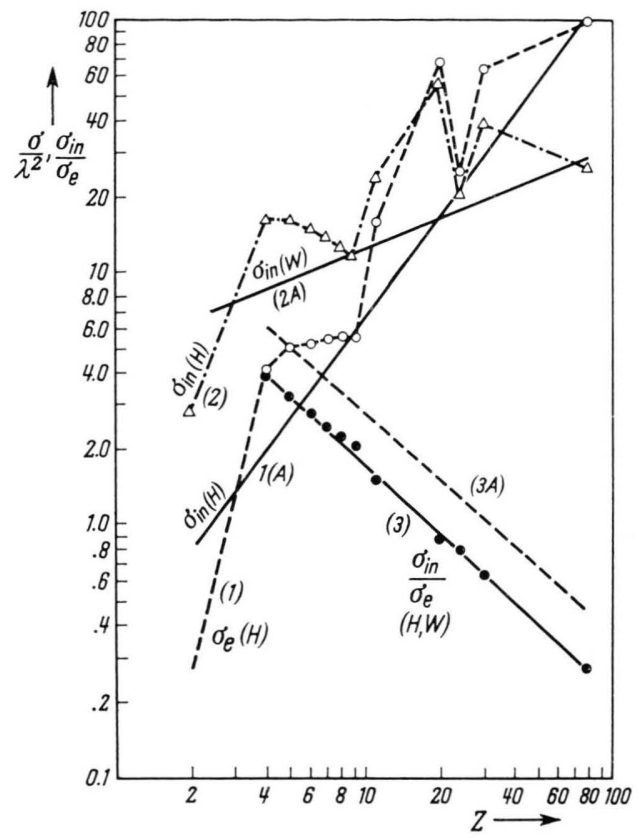

Fig. 3. Dependence of total elastic and inelastic scattering cross-sections of atoms on $Z$; curves 1 and 2 with $\mathrm{H}$ a $\mathrm{rt}$ re e radius, curves $1 \mathrm{~A}$ and $2 \mathrm{~A}$ with $\mathrm{W}$ e $\mathrm{ntz}$ el radius. Curve 3 denotes the ratio of $\frac{\text { Total inelastic cross-section }}{\text { Total elastic cross-section }}$ on both atom models; curve 3 A taken from Fig. 7 of Lenz's paper ${ }^{14}$.

Since for the evaluation of image contrast, the parameter of importance is $\int_{\alpha}^{\pi} \mathrm{d} \sigma=(\sigma)_{\alpha}^{\pi}$ i. e. the value of the cross-section for scattering beyond the effective objective angle $\alpha,(\sigma)_{\alpha}^{\pi}$ for carbon has been plotted in Fig. 4 for $50 \mathrm{kV}$ electrons against various values of aperture angles normally used in electron microscopy $\left(10^{-3}-3 \cdot 10^{-2}\right.$ radian $)$. Both the elastic $\left(\sigma_{\mathrm{e}}\right)$ as well as the inelastic $\left(\sigma_{\text {in }}\right)$ cross-sections, as well as the total cross-section i. e. $\sigma_{\mathrm{e}}+\sigma_{\text {in }}$ are shown in Fig. 4, calculated on both $\mathrm{H}$ a r tree and Wentzel radius basis. In Fig. 5 is represented the scattering crosssection per gm., $S$, of various substances for two aperture angles viz. $3.5 \times 10^{-3}$, and $10^{-2}$ radians, for $50 \mathrm{kV}$ electrons, $S$ being given by

$$
S=\frac{N_{\mathrm{A}}}{A} \cdot\left(\sigma_{\mathrm{e}}+\sigma_{\mathrm{in}}\right)
$$

where $A=$ At. wt., $N_{\mathrm{A}}=$ Avogadro's No. 


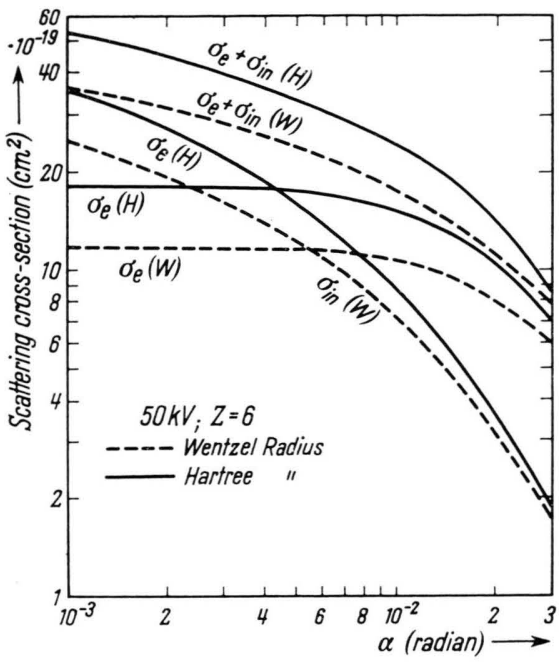

Fig. 4. Angular variation of elastic and inelastic scattering cross-section of carbon atom for $50 \mathrm{kV}$ electrons.

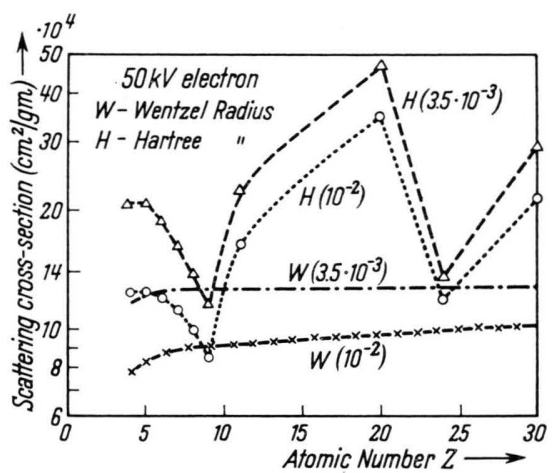

Fig. 5. Variation of scattering cross-section per gm. of various elements with Atomic Number $Z$.

26 M. L. De, Nature [London] 192, 547 [1961].

\section{Conclusion}

From the figures reproduced above one finds that, owing to the regular nature of variation of the W e n t z e l radius with $Z$ (Fig. 1, curve a), the corresponding scattering cross-section also show a continuous variation with atomic number [Fig. 3 , lines $1(\mathrm{~A})$ and $2(\mathrm{~A})]$, while the total scattering crosssection per gm. $S$ is remarkably constant that is independent of variation in $Z$ (Fig. 5, curves w). But it has been shown ${ }^{26}$ already that if one uses the Lenz's equation with Wentzel radius, the calculated values of scattering cross-section do not agree satisfactorily with values obtained from experimental results.

On the other hand, due to the irregular variation of the $\mathrm{Hartree}$ radius with $Z$ (Fig. 1, curve b) there is discontinuous variation with $Z$ of the scattering cross-section per atom (Fig. 3 , curves 1,2 ) ; also the total cross-section per gm. $S$, is seen to vary with $Z$ rather irregularly (Fig. 5, curve $\mathrm{H}$ ). This means that the image contrast for the same amount of object matter is liable to vary with atomic number.

The same comclusion is also reached from the experimental standpoint as has been shown by the author in a recent communication ${ }^{27}$.

The author thanks Prof. N. N. DAs Gupta of Calcutta University for his kind interest and also the Ministry of Scientific Research and Cultural Affairs for financial support.

27 M. L. DE, Naturwissenschaften 49, 296 [1962]. 